\title{
FINITE PRODUCTS OF REGULARIZED PRODUCTS
}

\author{
Francisco Diaz y Diaz and Eduardo Friedman
}

ABSTRACT. The product $\left(\widehat{\prod}_{m} a_{m}\right) \cdot\left(\widehat{\prod}_{m} b_{m}\right)$ of two regularized products is not in general equal to the regularized product $\widehat{\prod}_{m}\left(a_{m} \cdot b_{m}\right)$. We consider the discrepancy $F$, defined by

$$
\exp (F):=\frac{\widehat{\prod}_{m}\left(a_{m} \cdot b_{m}\right)}{\left(\widehat{\prod}_{m} a_{m}\right) \cdot\left(\widehat{\prod}_{m} b_{m}\right)} .
$$

When the terms $a_{m}$ and $b_{m}$ depend on parameters, we show in certain cases that $F$ is a polynomial in these parameters.

\section{Introduction}

The regularized product $\widehat{\prod}_{m} a_{m}$ of a countable set $\left\{a_{1}, a_{2}, \ldots\right\}$ of non-zero complex numbers is defined as

$$
\widehat{\prod}_{m} a_{m}:=\exp \left(-f^{\prime}(0)\right)
$$

where we assume that $f(s):=\sum_{m} a_{m}^{-s}$ converges in some right half-plane and has a meromorphic continuation to the $s$-plane which is regular at $s=0$, so that its derivative $f^{\prime}$ can be evaluated there. Several authors $[\mathrm{KW}][\mathrm{Mi}]$ have found examples where

$$
\left(\widehat{\prod_{m}} a_{m}\right) \cdot\left(\widehat{\prod}_{m} b_{m}\right)=\widehat{\prod_{m}}\left(a_{m} \cdot b_{m}\right),
$$

but Mizuno [Mi] has pointed out that this does not hold in general. ${ }^{1}$ For instance [FR, eq. (3.10)], if $z_{i}$ and $\tau_{i}(i=1,2)$ are positive real numbers, then

$$
\frac{\widehat{\prod}_{m=0}^{\infty}\left(m \tau_{1}+z_{1}\right) \cdot\left(m \tau_{2}+z_{2}\right)}{\left(\widehat{\prod}_{m=0}^{\infty}\left(m \tau_{1}+z_{1}\right)\right) \cdot\left(\widehat{\prod}_{m=0}^{\infty}\left(m \tau_{2}+z_{2}\right)\right)}=\exp \left(\frac{1}{2}\left(\frac{z_{1}}{\tau_{1}}-\frac{z_{2}}{\tau_{2}}\right) \log \left(\frac{\tau_{1}}{\tau_{2}}\right)\right) .
$$

A more complicated example was obtained by Mizuno [Mi, p. 157], namely,

$$
\frac{\widehat{\prod}_{l, m=0}^{\infty}\left(l \tau_{1}+m \eta_{1}+z_{1}\right) \cdot\left(l \tau_{2}+m \eta_{2}+z_{2}\right)}{\left(\widehat{\prod}_{l, m=0}^{\infty}\left(l \tau_{1}+m \eta_{1}+z_{1}\right)\right) \cdot\left(\widehat{\prod}_{l, m=0}^{\infty}\left(l \tau_{2}+m \eta_{2}+z_{2}\right)\right)}=: \exp (F),
$$

where

$$
F=\frac{\tau_{1} \eta_{2}-\tau_{2} \eta_{1}}{4}\left(\frac{\log \left(\frac{\eta_{2}}{\eta_{1}}\right)}{\eta_{1} \eta_{2}} B_{2}\left(\frac{z_{2} \eta_{1}-z_{1} \eta_{2}}{\tau_{2} \eta_{1}-\tau_{1} \eta_{2}}\right)-\frac{\log \left(\frac{\tau_{2}}{\tau_{1}}\right)}{\tau_{1} \tau_{2}} B_{2}\left(\frac{z_{2} \tau_{1}-z_{1} \tau_{2}}{\tau_{1} \eta_{2}-\tau_{2} \eta_{1}}\right)\right)
$$

Received by the editors January 9, 2007.

2000 Mathematics Subject Classification. Primary 11M36; Secondary 11M41.

Key words and phrases. Regularized products, Dirichlet series.

This work was partially supported by Chilean FONDECYT grants 1040585 and 7060247.

${ }^{1}$ We always assume that logarithm branches are chosen so that $\left(a_{m} b_{m}\right)^{-s}=a_{m}^{-s} b_{m}^{-s}$. 
$B_{2}(x):=x^{2}-x+\frac{1}{6}$, all parameters $z_{i}, \tau_{i}$ and $\eta_{i}$ are again assumed real and positive, and $\tau_{1} \eta_{2}-\tau_{2} \eta_{1} \neq 0$.

Shintani [Sh, pp. 204, 206] had earlier considered a related example

$$
\frac{\widehat{\prod}_{\mathbf{m} \in \mathbb{N}_{0}^{r}} \prod_{j=1}^{n} L_{j}(\mathbf{y}+\mathbf{m})}{\prod_{j=1}^{n} \widehat{\prod}_{\mathbf{m} \in \mathbb{N}_{0}^{r}} L_{j}(\mathbf{y}+\mathbf{m})}=: \mathrm{e}^{F(\mathbf{y})} .
$$

Here $L_{1}, L_{2}, \ldots, L_{n}$ are $n$ linear forms with positive coefficients in $r$ positive variables $\mathbf{y}=\left(y_{1}, \ldots, y_{r}\right)$ and $\mathbb{N}_{0}:=\mathbb{N} \cup\{0\}$, where $\mathbb{N}$ denotes the positive integers. Shintani showed that $F$ is a polynomial in $\mathbf{y}$ of degree at most $r$ and used this to study the derivative at $s=0$ of $L$-functions $L(s, \chi)$ attached to Hecke characters $\chi$ of a totally real number field. In this case $L_{j}$ is a real embedding of a linear form with coefficients in the number field and $\prod_{j} L_{j}$ is the norm form.

Given a regularized product $\widehat{\prod}_{m}\left(\prod_{j=1}^{n} a_{m}^{(j)}\right)$ of terms that factor in some natural way, we may compare it with the product of the individual regularized products $\widehat{\prod}_{m} a_{m}^{(j)}$ (when these are defined). Mizuno suggests studying the discrepancy $F$, defined by

$$
\mathrm{e}^{F}:=\frac{\widehat{\prod}_{m} \prod_{j=1}^{n} a_{m}^{(j)}}{\prod_{j=1}^{n} \widehat{\prod}_{m} a_{m}^{(j)}},
$$

as it seems that $F$ is often far simpler than the regularized products themselves. In (1) this is certainly born out since the regularized products involved are essentially Barnes' double $\Gamma$-functions. The discrepancy $F$ in (2), on the other hand, is just a polynomial in the constant terms $z_{i}$ of the regularized product, and a somewhat more complicated function of the coefficients $\tau_{i}$ and $\eta_{i}$ of the terms in degree one.

As the few known examples of the discrepancy $F$ only involve products of terms of degree one in $m$, here we consider products of general polynomials. However, in order to ensure the existence of the regularized products we must make some assumptions on the polynomials. Several authors [Ma] [Ca2] [Sa] [Li] have given conditions on the polynomial $P$ guaranteeing the existence of a meromorphic continuation in $s$ of the Dirichlet series $\sum_{\mathbf{m} \in \mathbb{N}^{r}} P(\mathbf{m})^{-s}$, or more generally of $\sum_{\mathbf{m} \in \mathbb{N}^{r}} \varphi(\mathbf{m}) P(\mathbf{m})^{-s}$, where $\varphi(\mathbf{m})$ is an arbitrary complex polynomial. We choose Mahler's conditions, as they are simple to state and imply that a meromorphic continuation of the series to the whole $s$-plane exists and is regular at $s=0$.

Mahler's hypothesis on $P$ [Ma, p. 385, Klasse A]. $P(\mathbf{x})=P\left(x_{1}, x_{2}, \ldots, x_{r}\right) \in \mathbb{C}[\mathbf{x}]$ does not vanish anywhere in the closed real first "octant" $x_{i} \geq 0(1 \leq i \leq r)$. Its homogeneous part of highest degree $P_{\text {top }}(\mathbf{x})$ is not constant and vanishes nowhere in the closed real first octant, except for $P_{\text {top }}(\mathbf{0})=0$.

Notice that if $P_{j}(\mathbf{x})$ satisfies this assumption for $1 \leq j \leq n$, then so does $\prod_{j=1}^{n} P_{j}(\mathbf{x})$. Under Mahler's hypothesis on $P_{j}$, we can choose for each $j$ a continuous branch of $\log P_{j}(\mathbf{x})$ for real $\mathbf{x}$ in the first octant, this choice being unique up to adding a fixed single multiple of $2 \pi i$. Having fixed these branches for each $j$, we define $\log \prod_{j=1}^{n} P_{j}(\mathbf{x}):=\sum_{j=1}^{n} \log P_{j}(\mathbf{x})$. 
Theorem 1. Let $P_{j}(\mathbf{x}) \in \mathbb{C}[\mathbf{x}]$ be $n$ complex polynomials in $r$ variables, all satisfying Mahler's hypothesis above, and define $F=F\left(P_{1}, P_{2}, \ldots, P_{n}\right)$ by

$$
\mathrm{e}^{F}:=\frac{\widehat{\prod}_{\mathbf{m} \in \mathbb{N}^{r}} \prod_{j=1}^{n} P_{j}(\mathbf{m})}{\prod_{j=1}^{n} \widehat{\prod}_{\mathbf{m} \in \mathbb{N}^{r}} P_{j}(\mathbf{m})} .
$$

Then $F$ is a polynomial of degree at most $r$ in the coefficients of the $P_{j}$ of non-maximal degree.

In other words, if we decompose $P_{j}(\mathbf{x})=P_{j \text {,top }}(\mathbf{x})+\sum_{I_{j}} a_{I_{j}} \mathbf{x}^{I_{j}}$, where the degree $\left|I_{j}\right|$ of the multi-indices $I_{j}$ is strictly less than the degree of $P_{j}$, then $F$ is a polynomial of degree at most $r$ in the $a_{I_{j}}(1 \leq j \leq n)$. We note that the logarithm branch for $F$ in (5) is clear, as it is obtained directly from the Dirichlet series defining the regularized products (see (8)).

Shintani and Mizuno's examples cited above show that $F$ in (5) is indeed not a polynomial in the coefficients of the top-degree forms $P_{j, \text { top }}$. Our proof, based on [FR, $\S 3]$, sheds no light on the dependence of $F$ on these coefficients and yields surprisingly little about $F$. Rather than compute $F$ explicitly, we show that it is a polynomial by proving the vanishing of all sufficiently high-order partial derivatives.

A direct corollary of Theorem 1 is a generalization to higher-degree polynomials of Shintani's result on products of linear forms (3).

Corollary 2. Fix $n$ polynomials $P_{j}(\mathbf{x}) \in \mathbb{C}[\mathbf{x}]$ in $r$ variables as above. For real $y_{i} \geq 0 \quad(1 \leq i \leq r)$, let $F(\mathbf{y})=F\left(y_{1}, \ldots, y_{r}\right)$ be defined by

$$
\mathrm{e}^{F(\mathbf{y})}:=\frac{\widehat{\prod}_{\mathbf{m} \in \mathbb{N}^{n}} \prod_{j=1}^{n} P_{j}(\mathbf{y}+\mathbf{m})}{\prod_{j=1}^{n} \widehat{\prod}_{\mathbf{m} \in \mathbb{N}^{r}} P_{j}(\mathbf{y}+\mathbf{m})} .
$$

Then $F$ is a polynomial in $\mathbf{y}=\left(y_{1}, \ldots, y_{r}\right)$ of degree at most $r$.

This follows since $P_{j}(\mathbf{y}+\mathbf{x})$ has the same top-degree form in $\mathbf{x}$ as $P_{j}(\mathbf{x})$.

We can also treat the Hurwitz form of a regularized product [JL, p. 1].

Corollary 3. Fix $n$ real polynomials $P_{j}(\mathbf{x})$ in $r$ variables, all satisfying Mahler's hypothesis and having non-negative coefficients. For real $z_{j} \geq 0(1 \leq j \leq n)$, let

$$
\mathrm{e}^{F(\mathbf{z})}:=\frac{\widehat{\prod}_{\mathbf{m} \in \mathbb{N}^{r}} \prod_{j=1}^{n}\left(z_{j}+P_{j}(\mathbf{m})\right)}{\prod_{j=1}^{n} \widehat{\prod}_{\mathbf{m} \in \mathbb{N}^{r}}\left(z_{j}+P_{j}(\mathbf{m})\right)} .
$$

Then $F$ is a polynomial in $\mathbf{z}=\left(z_{1}, \ldots, z_{n}\right)$ of degree at most $r$.

We will give in $\S 2$ a sharper bound on the degree of $F(\mathbf{z})$. In particular, when $\operatorname{deg}\left(P_{j}\right)>r(1 \leq j \leq n)$, we will show that $F$ depends on the polynomials $P_{j}$, but not on $\mathbf{z}$.

In $\S 2$ we state and prove a slightly more general form of Theorem 1, where we allow polynomial powers $\varphi(\mathbf{m})$ and show that $F$ in

$$
\mathrm{e}^{F}:=\frac{\widehat{\prod}_{\mathbf{m} \in \mathbb{N}^{r}}\left(\prod_{j=1}^{n} P_{j}(\mathbf{m})\right)^{\varphi(\mathbf{m})}}{\prod_{j=1}^{n} \widehat{\prod}_{\mathbf{m} \in \mathbb{N}^{r}}\left(P_{j}(\mathbf{m})^{\varphi(\mathbf{m})}\right)}
$$

also satisfies the conclusion of Theorem 1, with the bound $r$ on the degree of the polynomial replaced by $r+\operatorname{deg}(\varphi)$. In $\S 3$ we list some formal properties of $F$. 


\section{Proof of Theorem 1}

We first describe Mahler's results concerning the meromorphic continuation of Dirichlet series of the form

$$
Z(s)=Z(s ; \log P, \varphi):=\sum_{\mathbf{m} \in \mathbb{N}^{r}} \frac{\varphi(\mathbf{m})}{P(\mathbf{m})^{s}},
$$

where $\varphi$ is an arbitrary complex polynomial in $r$-variables and $P$ satisfies Mahler's hypothesis (see $\S 1$ ). Since we have assumed that $P(\mathbf{x}) \neq 0$ for all $\mathbf{x}=\left(x_{1}, \ldots, x_{r}\right)$ in the (real) first octant $x_{i} \geq 0 \quad(1 \leq i \leq r)$, a continuous branch of $\log P(\mathbf{x})$ can be chosen in this simply connected region [Ma, §3]. To define $Z(s)$ we fix such a branch. Note that any two continuous branches differ by a continuous discrete-valued function, and so must differ by a fixed multiple of $2 \pi i$.

Mahler showed [Ma, pp. 397-398, Satz II] that the series (5) converges absolutely and uniformly in compact subsets of the right half-plane defined by

$$
\operatorname{Re}(s) \operatorname{deg}(P)-\operatorname{deg}(\varphi)>r,
$$

and that $Z(s)$ has a meromorphic continuation to all of $\mathbb{C}$, regular at $s=0$. Mahler's proof also yields that $Z(s)$ is analytic in the coefficients of $P$ in a small enough neighborhood (in coefficient-space) of $P$. The point here is that a branch of $\log P(\mathbf{x})$ on the entire first octant can be chosen locally analytically in coefficient-space.

We shall need the following computation, readily proved by induction on $k$.

Lemma. Write the polynomial $P$ in $r$ variables $\mathbf{x}=\left(x_{1}, \ldots x_{r}\right)$ as $P(\mathbf{x})=\sum_{I} a_{I} \mathbf{x}^{I}$, where $I=\left(I_{1}, \ldots, I_{r}\right)$ runs over distinct multi-indices, $a_{I} \in \mathbb{C}$ and $\mathbf{x}^{I}:=\prod_{i=1}^{r} x_{i}^{I_{i}}$.

Let $\mathcal{D}=\frac{\partial^{k}}{\partial a_{I^{(1)}} \partial a_{I^{(2)}} \cdots \partial a_{I^{(k)}}}$ be a differential operator consisting of $k$ successive partial derivatives with respect to any sequence $a_{I^{(1)}}, a_{I^{(2)}}, \ldots, a_{I^{(k)}}$ of coefficients of $P$. Then

$$
\mathcal{D}\left(P(\mathbf{x})^{-s}\right)=(-1)^{k}\left(\prod_{p=0}^{k-1}(s+p)\right) \mathbf{x}^{\sum_{p=1}^{k} I^{(p)}} P(\mathbf{x})^{-s-k} .
$$

We now prove the following generalization of Theorem 1, which we state in terms of Dirichlet series rather than regularized products.

Theorem 4. Let $P_{j}(\mathbf{x}) \in \mathbb{C}[\mathbf{x}]$ be $n$ complex polynomials in $r$ variables, all satisfying Mahler's hypothesis above, and let $\varphi(\mathbf{x}) \in \mathbb{C}[\mathbf{x}]$ be any polynomial in $\mathbf{x}$. Define for $\operatorname{Re}(s) \gg 0$,

$$
f_{j}(s):=\sum_{\mathbf{m} \in \mathbb{N}^{r}} \frac{\varphi(\mathbf{m})}{P_{j}(\mathbf{m})^{s}} \quad(1 \leq j \leq n), \quad f_{0}(s):=\sum_{\mathbf{m} \in \mathbb{N}^{r}} \frac{\varphi(\mathbf{m})}{\prod_{j=1}^{n} P_{j}(\mathbf{m})^{s}},
$$

and, after analytically continuing the $f_{j}(0 \leq j \leq n)$,

$$
F:=-f_{0}^{\prime}(0)+\sum_{j=1}^{n} f_{j}^{\prime}(0) .
$$

Then $F$ is a polynomial of degree at most $r+\operatorname{deg}(\varphi)$ in the coefficients of the $P_{j}$ of non-maximal degree.

Note that $F$, and even each $f_{j}(s)$, depends linearly on $\varphi$, so we ignore this dependence in the above theorem. 
Proof. We shall actually prove a more precise bound on the degree of $F$. Namely,

Claim. Given $n$ non-negative integers $\ell_{j}<\operatorname{deg}\left(P_{j}\right)$, consider $F$ as a function of just the $a_{I^{(j)}}$ appearing in the $P_{j}(\mathbf{x}) \quad(1 \leq j \leq n)$ as coefficients of terms $a_{I^{(j)}} \mathbf{x}^{I^{(j)}}$ having degree at most $\ell_{j}$ in $\mathbf{x}$. Then, as a polynomial in these $a_{I^{(j)}}(1 \leq j \leq n)$, the degree of $F$ does not exceed $\max _{1 \leq j \leq n}\left\{\left\lfloor\frac{r+\operatorname{deg}(\varphi)}{\operatorname{deg}\left(P_{j}\right)-\ell_{j}}\right\rfloor\right\}$.

Here, $\lfloor t\rfloor$ is the integer such that $\lfloor t\rfloor \leq t<\lfloor t\rfloor+1$.

To prove the Claim, let

$$
\mathfrak{D}=\mathcal{D}_{1} \mathcal{D}_{2} \cdots \mathcal{D}_{n}, \quad \mathcal{D}_{j}=\frac{\partial^{k_{j}}}{\partial a_{I^{(j, 1)}} \partial a_{I^{(j, 2)}} \cdots \partial a_{I^{\left(j, k_{j}\right)}}},
$$

be the composition of $n$ commuting differential operators $\mathcal{D}_{j}$, each involving only coefficients $a_{I^{(j, p)}}$ of $P_{j}$ in degree at most $\ell_{j}$. Our Claim amounts to showing that if the order $k=\sum_{j=1}^{n} k_{j}$ of $\mathfrak{D}$ satisfies

$$
k>\max _{1 \leq j \leq n}\left\{\frac{r+\operatorname{deg}(\varphi)}{\operatorname{deg}\left(P_{j}\right)-\ell_{j}}\right\},
$$

then $\mathfrak{D}(F)=0$ identically. For the remainder of this proof we assume (9).

We first prove the formula for $1 \leq j \leq n$,

$$
\left.\frac{\partial}{\partial s}\right|_{s=0} \mathfrak{D}\left(f_{j}(s)\right)= \begin{cases}(-1)^{k_{j}}\left(k_{j}-1\right) ! \sum_{\mathbf{m} \in \mathbb{N}^{r}} \varphi(\mathbf{m}) \mathbf{m}^{\sum_{p=1}^{k_{j}} I^{(j, p)}} P_{j}(\mathbf{m})^{-k_{j}} & \text { if } \mathfrak{D}=\mathcal{D}_{j} ; \\ 0, & \text { otherwise }\end{cases}
$$

where we shall presently see that the Dirichlet series on the right converges absolutely.

If $\mathfrak{D} \neq \mathcal{D}_{j}$, so that $\mathfrak{D}$ involves derivatives with respect to coefficients of some $P_{j^{\prime}}$ $\left(j \neq j^{\prime}\right)$, then $\mathfrak{D}\left(f_{j}(s)\right)=0$ since $f_{j}$ depends only on $P_{j}$. In proving (10) we may therefore assume $\mathfrak{D}=\mathcal{D}_{j}$, so that $k_{j}=k>\frac{r+\operatorname{deg}(\varphi)}{\operatorname{deg}\left(P_{j}\right)-\ell_{j}}$.

Take $\operatorname{Re}(s) \gg 0$ and apply the Lemma to obtain

$$
\mathcal{D}_{j}\left(f_{j}(s)\right)=\left((-1)^{k_{j}} \prod_{p=0}^{k_{j}-1}(s+p)\right) \sum_{\mathbf{m} \in \mathbb{N}^{r}} \varphi(\mathbf{m}) \mathbf{m}^{\sum_{p=1}^{k_{j}} I^{(j, p)}} P_{j}(\mathbf{m})^{-s-k_{j}} .
$$

To check where the above series converges, note that

$$
\begin{aligned}
\left(k_{j}+\operatorname{Re}(s)\right) \operatorname{deg}\left(P_{j}\right)- & \operatorname{deg}(\varphi)-\sum_{p=1}^{k_{j}}\left|I^{(j, p)}\right| \\
& \geq-\operatorname{deg}(\varphi)-\ell_{j} k_{j}+\left(k_{j}+\operatorname{Re}(s)\right) \operatorname{deg}\left(P_{j}\right) \\
& =-\operatorname{deg}(\varphi)+k_{j}\left(\operatorname{deg}\left(P_{j}\right)-\ell_{j}\right)+\operatorname{Re}(s) \operatorname{deg}\left(P_{j}\right),
\end{aligned}
$$

since we have assumed that $\mathcal{D}_{j}$ involves only coefficients of terms $x^{I}$ of degree $|I|$ at most $\ell_{j}$. By (6) and (12), the right-hand side of (11) converges and gives an analytic function of $s$, provided

$$
k_{j}\left(\operatorname{deg}\left(P_{j}\right)-\ell_{j}\right)+\operatorname{Re}(s) \operatorname{deg}\left(P_{j}\right)>r+\operatorname{deg}(\varphi) .
$$


In particular, since $k_{j}>\frac{r+\operatorname{deg}(\varphi)}{\operatorname{deg}\left(P_{j}\right)-\ell_{j}}$, we find that right-hand side of $(11)$ converges absolutely in an open right half-plane containing $s=0$. Thus, although (11) was derived for $\operatorname{Re}(s)$ large enough, by analytic continuation it also holds at $s=0$. We may therefore differentiate both sides of (11) with respect to $s$ and set $s=0$ to obtain $(10)$.

To state the next formula it will be convenient to call $\mathfrak{D}$ pure if $\mathfrak{D}=\mathcal{D}_{j}$ for some $j(1 \leq j \leq n)$. Otherwise we will call $\mathfrak{D}$ mixed. We now prove

$$
\left.\frac{\partial}{\partial s}\right|_{s=0} \mathfrak{D}\left(f_{0}(s)\right)= \begin{cases}0, & \text { if } \mathcal{D} \text { is mixed } \\ \left.\frac{\partial}{\partial s}\right|_{s=0} \mathfrak{D}\left(f_{j}(s)\right) & \text { if } \mathfrak{D}=\mathcal{D}_{j}\end{cases}
$$

As before, the Lemma gives for $\operatorname{Re}(s) \gg 0$,

$$
\mathfrak{D}\left(f_{0}(s)\right)=(-1)^{k}\left(\prod_{j=1}^{n} \prod_{p=0}^{k_{j}-1}(s+p)\right) \sum_{\mathbf{m} \in \mathbb{N}^{r}} \varphi(\mathbf{m}) \mathbf{m}^{\sum_{j=1}^{n} \sum_{p=1}^{k_{j}} I^{(j, p)}} \prod_{j=1}^{n} P_{j}(\mathbf{m})^{-s-k_{j}} .
$$

Note that here we have used our choice of branch of $\log \prod_{j} P_{j}$.

For convergence of the Dirichlet series (14) we need

$$
\sum_{j=1}^{n}\left(\left(k_{j}+\operatorname{Re}(s)\right) \operatorname{deg}\left(P_{j}\right)-\sum_{p=1}^{k_{j}}\left|I^{(j, p)}\right|\right)>r+\operatorname{deg}(\varphi) .
$$

But $\left|I^{(j, p)}\right| \leq \ell_{j}$, so for convergence it suffices to ensure that

$$
\sum_{j=1}^{n} k_{j}\left(\operatorname{deg}\left(P_{j}\right)-\ell_{j}\right)+\operatorname{Re}(s) \sum_{j=1}^{n} \operatorname{deg}\left(P_{j}\right)>r+\operatorname{deg}(\varphi) .
$$

Setting $s=0$ on the left-hand side above, we calculate

$$
\begin{aligned}
\sum_{j=1}^{n} k_{j}\left(\operatorname{deg}\left(P_{j}\right)-\ell_{j}\right) & \geq \sum_{j=1}^{n} k_{j} \min _{1 \leq j \leq n}\left\{\operatorname{deg}\left(P_{j}\right)-\ell_{j}\right\} \\
& =k \min _{1 \leq j \leq n}\left\{\operatorname{deg}\left(P_{j}\right)-\ell_{j}\right\}>r+\operatorname{deg}(\varphi),
\end{aligned}
$$

by (9). The series in (14) thus converges in an open neighborhood of $s=0$, so (14) holds there.

We can now conclude the proof of (13). If $\mathfrak{D}$ is mixed, then $\prod_{j=1}^{n} \prod_{p=0}^{k_{j}-1}(s+p)$ on the right-hand side of (14) includes a factor of $s^{2}$. We then have the trivial vanishing $\left.\frac{\partial}{\partial s}\right|_{s=0} \mathfrak{D}\left(f_{0}(s)\right)=0$. If $\mathfrak{D}=\mathcal{D}_{j}$ is pure, we have only a single factor of $s$. From (14) we then find, using $\mathfrak{D}=\mathcal{D}_{j}$ and $k=k_{j}$,

$$
\left.\frac{\partial}{\partial s}\right|_{s=0} \mathfrak{D}\left(f_{0}(s)\right)=(-1)^{k_{j}}\left(k_{j}-1\right) ! \sum_{\mathbf{m} \in \mathbb{N}^{r}} \varphi(\mathbf{m}) \mathbf{m}^{\sum_{p=1}^{k_{j}} I^{(j, p)}} P_{j}(\mathbf{m})^{-k_{j}}=\left.\frac{\partial}{\partial s}\right|_{s=0} \mathfrak{D}\left(f_{j}(s)\right),
$$

by $(10)$.

Having now established (10) and (13), we deduce

$$
\mathfrak{D}(F)=\mathfrak{D}\left(-f_{0}^{\prime}(s)+\sum_{j=1}^{n} f_{j}^{\prime}(s)\right)=0,
$$


as claimed after (9).

We can sharpen Corollary 3 by taking all $\ell_{j}=0$ in the Claim above.

Corollary 5. Fix $n$ real polynomials $P_{j}(\mathbf{x})$ in $r$ variables, all satisfying Mahler's hypothesis and having non-negative coefficients. For $\operatorname{Re}\left(z_{j}\right)>0(1 \leq j \leq n)$ and $\operatorname{Re}(s) \gg 0$, define

$$
f_{j}(s):=\sum_{\mathbf{m} \in \mathbb{N}^{r}} \varphi(\mathbf{m})\left(z_{j}+P_{j}(\mathbf{m})\right)^{-s}, \quad f_{0}(s):=\sum_{\mathbf{m} \in \mathbb{N}^{r}} \varphi(\mathbf{m}) \prod_{j=1}^{n}\left(z_{j}+P_{j}(\mathbf{m})\right)^{-s},
$$

and,

$$
F:=-f_{0}^{\prime}(0)+\sum_{j=1}^{n} f_{j}^{\prime}(0)
$$

Then $F$ is a polynomial in the $z_{j}$ of degree at most $\max _{1 \leq j \leq n}\left\{\left\lfloor\frac{r+\operatorname{deg}(\varphi)}{\operatorname{deg}\left(P_{j}\right)}\right\rfloor\right\}$.

In particular, $F$ is independent of $\mathbf{z}$ if $\varphi$ is constant and if all the $P_{j}$ are of degree at least $r+1$.

We conclude this section with some remarks on Theorem 4.

1. Examination of the proof shows that the conclusion of Theorem 4 still holds if in the definition of $f_{j}(s)(0 \leq j \leq n)$ the $\operatorname{sum} \sum_{\mathbf{m} \in \mathbb{N}^{r}} \varphi(\mathbf{m}) P_{j}(\mathbf{m})^{-s}$ is replaced by the integral $\int_{\mathbf{x} \in \mathbb{R}_{+}^{r}} \varphi(\mathbf{x}) P_{j}(\mathbf{x})^{-s} d \mathbf{x}$. Here $P_{0}(\mathbf{x}):=\prod_{j=1}^{n} P_{j}(\mathbf{x})$. The only additional point needed in the proof is Mahler's result [Ma, p. 392, Satz I] showing the convergence and meromorphic continuation of these integrals (still assuming Mahler's hypothesis for each $P_{j}$, of course).

2. We have assumed that the polynomials $P_{j}$ satisfy Mahler's hypothesis, but the formal nature of our proof shows that what matters is that the Dirichlet series $f_{j}(s)$ is defined, converges absolutely for $\operatorname{Re}(s) \operatorname{deg}\left(P_{j}\right)-\operatorname{deg}(\varphi)>r$, and analytically extends to a function regular at $s=0$. As we mentioned in $\S 1$, other authors have found alternative hypotheses that guarantee this.

Even the polynomial nature of the $P_{j}$ or $\varphi$ is not essential, as we could consider series of the form

$$
f_{j}(s)=\sum_{\mathbf{m}} \varphi(\mathbf{m}) P_{j}\left(\mathbf{m}, \mathbf{a}_{\mathbf{j}}\right)^{-s},
$$

where $P_{j}$ depends on some parameter $\mathbf{a}_{\mathbf{j}}$ ranging over some open subset of some Euclidean space, and $\mathbf{m}$ runs over a countable set. Aside from the obviously necessary convergence for $\operatorname{Re}(s) \gg 0$ and the existence of a meromorphic continuation in $s$ of $f_{j}(s)(0 \leq j \leq n)$, what matters in the proof of Theorem 4 is that all sufficiently highorder derivatives $\mathcal{D}_{j} P_{j}\left(\mathbf{m}, \mathbf{a}_{\mathbf{j}}\right)^{-s}$ (taken with respect to $\mathbf{a}_{\mathbf{j}}$ ) decrease quickly enough with $\mathbf{m}$ for $\left.\sum_{\mathbf{m}} \varphi(\mathbf{m}) \mathcal{D}_{j} P_{j}\left(\mathbf{m}, \mathbf{a}_{\mathbf{j}}\right)^{-s}\right|_{s=0}$ to converge.

3. Our proof yields that the values $f_{j}(0)$, and more generally the values $f_{j}(-N)$ for each fixed non-negative integer $N$, are also polynomial functions of the coefficients of $P_{j}$ of non-maximal degree $(1 \leq j \leq n)$. To see this it suffices to take $k_{j}>$ $\frac{N \operatorname{deg}\left(P_{j}\right)+r+\operatorname{deg} \varphi}{\operatorname{deg}\left(P_{j}\right)-\ell_{j}}$ in (11) and set $s=-N$. Thus, for fixed $j, \ell_{j}<\operatorname{deg}\left(P_{j}\right)$ and $N$, considered as a function of just the $a_{I^{(j)}}$ appearing in $P_{j}(\mathbf{x})$ as coefficients of 
terms $a_{I^{(j)}} \mathbf{x}^{I^{(j)}}$ having degree at most $\ell_{j}, f_{j}(-N)$ is a polynomial of degree not exceeding $\left\{\left\lfloor\frac{N \operatorname{deg}\left(P_{j}\right)+r+\operatorname{deg}(\varphi)}{\operatorname{deg}\left(P_{j}\right)-\ell_{j}}\right\rfloor\right\}$. For a detailed study of $f_{j}(-N)$ for certain classes of polynomials see [Ca1] [Ca2].

4. The discrepancy $F$ in

$$
\mathrm{e}^{F}:=\frac{\widehat{\prod}_{m} \prod_{j=1}^{n} a_{m}^{(j)}}{\prod_{j=1}^{n} \widehat{\prod}_{m} a_{m}^{(j)}} .
$$

has the curious property of being unaltered by the omission of any finite number of indices $m$ from all the regularized products.

\section{Properties of $F$}

In this brief section we list some formal properties of $F$ in Theorem 4. To make these properties clearer we will write $F_{n}\left(P_{1}, \ldots, P_{n} ; \varphi\right)$ for $F$. One should bear in mind that $F$ also depends on the branches of $\log P_{j}$ used.

Proposition 6. $F$ has the following properties.

(a) Symmetry: $F_{n}\left(P_{1}, \ldots, P_{n} ; \varphi\right)$ is independent of the order of the $P_{j}$.

(b) Vanishing on the diagonal: $F_{n}(P, P, \ldots, P ; \varphi)=0$.

(c) Reduction of $n:$ For $n \geq 3$,

$$
F_{n}\left(P_{1}, P_{2}, \ldots, P_{n} ; \varphi\right)=F_{n-1}\left(P_{1} \cdot P_{2}, \ldots, P_{n} ; \varphi\right)+F_{2}\left(P_{1}, P_{2} ; \varphi\right) .
$$

(d) Reduction to two polynomials: For $n \geq 2$,

$$
F_{n}\left(P_{1}, P_{2}, \ldots, P_{n} ; \varphi\right)=\sum_{j=1}^{n-1} F_{2}\left(\prod_{k=1}^{j} P_{k}, P_{j+1} ; \varphi\right) .
$$

Proof. Property (a) is immediate from the definition of $F$ given in (8). If $P_{j}=P$ for $1 \leq j \leq n$, then $f_{0}(s)=f_{j}(n s)$. Property (b) then follows. To prove (c), observe that for $\operatorname{Re}(s) \gg 0$,

$$
\begin{aligned}
\sum_{j=1}^{n} & \sum_{\mathbf{m}} \varphi(\mathbf{m}) P_{j}(\mathbf{m})^{-s}-\sum_{\mathbf{m}} \varphi(\mathbf{m}) \prod_{j=1}^{n} P_{j}(\mathbf{m})^{-s} \\
= & \sum_{\mathbf{m}} \varphi(\mathbf{m})\left(P_{1}(\mathbf{m}) P_{2}(\mathbf{m})\right)^{-s}+\sum_{j=3}^{n} \sum_{\mathbf{m}} \varphi(\mathbf{m}) P_{j}(\mathbf{m})^{-s}-\sum_{\mathbf{m}} \varphi(\mathbf{m}) \prod_{j=1}^{n} P_{j}(\mathbf{m})^{-s} \\
& +\sum_{j=1}^{2} \sum_{\mathbf{m}} \varphi(\mathbf{m}) P_{j}(\mathbf{m})^{-s}-\sum_{\mathbf{m}} \varphi(\mathbf{m})\left(P_{1}(\mathbf{m}) P_{2}(\mathbf{m})\right)^{-s}
\end{aligned}
$$

where we have used our convention that logarithm branches are always chosen for products so that $\left(P_{1}(\mathbf{x}) P_{2}(\mathbf{x}) \cdots P_{k}(\mathbf{x})\right)^{-s}=P_{1}(\mathbf{x})^{-s} P_{2}(\mathbf{x})^{-s} \cdots P_{k}(\mathbf{x})^{-s}$ for $\mathbf{x}$ in the first octant. Property (c) follows from (13) by analytic continuation to $s=0$. Property (d) follows from (c) by induction on $n$. 


\section{References}

[Ca1] Pi. Cassou-Noguès, Séries de Dirichlet, Seminar on Number Theory, 1980-1981 (Talence, 1980-1981), Exp. No. 22, 14 pp., Univ. Bordeaux I.

[Ca2] _ Prolongement de certaines séries de Dirichlet, Amer. J. Math. 105 (1983) 13-58.

[FR] E. Friedman and S. Ruijsenaars, Shintani-Barnes zeta and gamma functions, Advances Math. 187 (2004) 362-395.

[JL] J. Jorgenson and S. Lang, Basic Analysis of Regularized Series and Products, Springer Lecture Notes in Math. 1425, Springer: Berlin, 1993.

[KW] N. Kurokawa and M. Wakayama, A generalization of Lerch's formula, Czechoslovak Math. J. 54 (2004) 941-947.

[Li] B. Lichtin, Generalized Dirichlet series and b-functions, Compositio Math. 65 (1988) 81-120.

[Ma] K. Mahler, Über einen Satz von Mellin, Math. Ann. 100 (1928) 384-398.

[Mi] Y. Mizuno, Generalized Lerch formulas: Examples of zeta-regularized products, J. Number Theory 118 (2006) 155-171.

[Sa] P. Sargos, Prolongement méromorphe des séries de Dirichlet associées à des fractions rationnelles de plusieurs variables, Ann. Inst. Fourier 34 (1984) 83-123.

[Sh] T. Shintani, On values at $s=1$ of certain L functions of totally real algebraic number fields, in: Algebraic Number Theory, Papers Contributed for the International Symposium, Kyoto 1976 (S. Iyanaga, Ed.), Japan Society for the Promotion of Science, Tokyo 1977, 201-212.

Institut de Mathématiques, Université Bordeaux I, 351 Cours de la Libération, F33405 Talence CÉdex, France

E-mail address: diaz@math.u-bordeaux1.fr

Departamento de Matemática, Universidad de Chile, Casilla 653, Santiago 1, Chile

E-mail address: friedman@uchile.cl 